\title{
Empirical Mode Decomposition and the two-tone separation problem in the presence of noise
}

\author{
F.M. Ziaeyan Bahri ${ }^{\text {a }}$, J.J. Sharples ${ }^{\text {a }}$ \\ ${ }^{a}$ School of Physical, Environmental and Mathematical Sciences, UNSW Canberra, Australia \\ Email: f.bahri@adfa.edu.au
}

\begin{abstract}
The Empirical Mode Decomposition (EMD) is a decomposition method that provides an alternative way to analyse nonlinear and non-stationary time series. Since its inception in the late 1990s, EMD has been applied in a number of areas, including bio-medicine, neuroscience, epidemiology, chemical engineering, finance, at-mospheric turbulence, seismology and ocean dynamics. The EMD process decomposes a signal into intrinsic mode functions (IMFs); each IMF can have variable amplitude and tends to occupy a narrow spectral band-width, rather than having a single frequency. The question of how the EMD copes with time series with high spectral density therefore naturally arises, but has only attracted limited attention in the literature. A time series with high spectral density is one comprised of multiple signals each with very similar frequencies.

In this study we consider fundamental cases of synthetic time series composed of two sinusoidal signals with prescribed differences in their frequencies. Separating the two component signals from the input data is known as the 'two-tone separation problem'. We investigate the ability of the EMD method to successfully identify the two component signals as IMFs for each time series, and heuristic bounds on the utility of the EMD applied to high spectral density data are discussed.

In this study we consider two-tone signals without noise and find results similar to those already reported in the literature. We also consider noisy two-tone input signals and consider the ability of ensemble EMD (EEMD) to resolve the two component signals. This work appears to be the first to consider the two-tone separation problem in the presence of noise. Somewhat unsurprisingly, the addition of noise to the input data diminishes the ability of the EEMD method to identify and separate the two component signals. However, our results indicate that the presence of noise has a more prominent effect of the ability of EEMD to separate the two component signals than it does on its ability to distinguish the two-tone signal from a single waveform.

Expressing the results in terms of the ratio of the frequencies of the two component signals $f$, it was found that in the absence of noise EMD cannot distinguish a two-tone signal from a single waveform when $f>0.71$, and could only reliably separate the two component signals when $f<0.5$ approximately. These figures are in good agreement with those found in previous studies. For a noisy two-tone signal, it was found that EEMD was not able to distinguish the two-tone signal from a single waveform when $f>0.7$ and could only separate the two component signals when $f<0.4$ approximately.

In this initial study the effects of varying the amplitude of one component signal relative to the other was not considered. This would be an interesting extension of the research.
\end{abstract}

Keywords: Empirical mode decomposition, time series analysis, two-tone separation, frequency resolution 
F.M. Ziaeyan Bahri and J.J. Sharples, EMD and the two-tone separation problem with noise.

\section{INTRODUCTION}

Many methods of time series analysis, including signal processing techniques, are designed to be able to resolve separate component signals from data that are a superposition of a multitude of individual signals with different frequencies and amplitudes. Examples of such methods include the Fourier transform, wavelet analysis and the Empirical Mode Decomposition (Bracewell, 1965; Wickerhauser, 1994; Huang et al., 1998). While all such methods are known to be able to successfully resolve component signals in a broad range of applications, they all have their limitations. For example, the Fourier transform can perform poorly when confronted with nonlinear and non-stationary time series (Kolláth and Oláh, 2009). Empirical Mode Decomposition (EMD) was specifically developed to be able to accommodate data nonlinearity and non-stationarity, and has been shown to provide reliable and accurate results in several applications (Donnelly, 2006; Schlurmann, 2002). However, given the empirical underpinnings of EMD it is not possible to gain a comprehensive theoretical understanding of the limitations of the method when it comes to its ability to deal with particular idiosyncrasies in the data, such as abrupt non-stationarity, or high spectral density. As such, advances in our understanding of EMD are based on studies using synthetic times series, which have been constructed to investigate specific sensitivities of the method (Donnelly, 2006; Boudraa and Cexus, 2007).

Data with high spectral density is made up of distinct component signals, but with frequencies that are very similar. Obviously there are many applications where it is desirable to be able to distinguish the component signals, but this is complicated by the fact that when signals with very similar frequencies are combined they produce a 'beat effect'. In such situations, the data can present more as an amplitude modulated single frequency signal. To test the ability of a method to resolve separate frequency components, it is customary to consider the so-called 'two-tone separation' problem. In this problem, two sinusoidal signals of differing frequencies are superimposed and then the method is evaluated based on its ability to resolve the two separate signals.

The ability of EMD to resolve two signals with similar frequencies was studied by Rilling and Flandrin (2008), who used a simple synthetic data set made up of two sinusoidal signals. The amplitude ratio $a$ and the frequency ratio $f$ of the two component signals were varied systematically to identify a region in $(a, f)$-space where EMD could correctly separate the two-tone signal. Outside of this region, EMD either considered the two-tone signal as a single waveform or exhibited some other 'indeterminate' behaviour.

The work of Rilling and Flandrin (2008) was extended by Feldman (2009), who provided a theoretical analysis of the harmonic modes in a two-tone signal. Feldman (2009) also found that when $(a, f)$ fell within a certain region, EMD was able to resolve the two separate signals, but when $f \approx 1$ and $a \approx 0$ it was not possible for EMD to separate the two tones. For frequencies that were more dissimilar, EMD could resolve the two tones even when the amplitude ratio was small.

Other authors have also considered the two-tone separation problem using alternate data-driven methods such as synchrosqueezing (Wu et al., 2011), but in this paper will we maintain the focus on EMD. In all of the previous works mentioned here, the two-tone input signal was comprised of two 'pure' sinusoidal signals in the absence of noise. While this is a useful approach, inferences drawn from the results should be considered of limited validity to real-world cases, in which time series data is more often affected by noise. It is well known that in the presence of noise EMD can result in mode-mixing Huang and Wu (2008) and so it is of interest to understand how such effects might influence the ability of EMD to resolve two signals of similar frequency. In this study, we extend the approach taken in earlier studies by adding noise to a synthetic two-tone input signal. In this initial work only the sensitivity to frequency differences is considered. The sensitivity to differences in the amplitudes of the two component signals will form the subject of future work.

\section{Method AND DATA}

\subsection{EMD Method}

The EMD method is a data-adaptive technique for decomposing a time series into component series (Huang et al., 1998). Essentially, EMD applies a filtering technique based on upper and lower envelopes of the extreme points in a time series to iteratively extract zero-mean component time series, known as Intrinsic Mode Functions (IMFs) and a residual trend. Specifically, the IMFs satisfy the following defining conditions: (i) symmetric upper and lower envelopes (mean is equal to zero); (ii) the number of zero-crossings and extrema are either equal or differ by one. To obtain the IMFs, EMD implements the following algorithm:

1. Identify all the local extrema in the time series $y(t)$. 
F.M. Ziaeyan Bahri and J.J. Sharples, EMD and the two-tone separation problem with noise.

2. Interpolate the maxima and minima separately to define upper and lower envelopes.

3. Calculate the mean $m_{1}(t)$ of the upper and lower envelopes.

4. Calculate the first protomode as $h_{1}(t)=y(t)-m_{1}(t)$.

5. Reapply steps 1-4 until $h_{j}=h_{j-1}=h_{j-2}$, then $I M F_{1}(t)=h_{j}(t)$.

6. Calculate the residual $r_{1}(t)=y(t)-I M F_{1}(t)$, then reapply steps $1-5$ to $r_{1}(t)$ in place of $y(t)$ to deliver $I M F_{2}(t)$.

7. Calculate the residual $r_{2}(t)=r_{1}(t)-I M F_{2}(t)$, and repeat the above steps to obtain subsequent IMFs. The algorithm terminates when the number of zero-crossings and extrema of the residual are equal or at most differing by one. In this case the residual is interpreted as the trend in the original series $y(t)$,

After obtaining all the IMFs, the time series can be rewritten as:

$$
y(t)=\sum_{j=1}^{n} I M F_{j}(t)+r_{n}(t),
$$

where $r_{n}(t)$ is the residual/trend. For a more detailed exposition of the EMD procedure the reader is referred to Huang and $\mathrm{Wu}(2008)$.

As mentioned already, noisy input data can result in mode-mixing across the IMFs. To circumvent this issue when dealing with noisy time series, the EMD procedure is implemented as part of an ensemble process, which is referred to as ensemble EMD (EEMD). In the EEMD procedure, random noise $(\epsilon \sim N(0, \delta))$ is added to the input data before the EMD procedure is applied. The process of adding random noise to the signal and applying EMD is repeated a large number of times and the ensemble average of the IMFs found from these numerous EMD applications define the final ensemble IMFs (Huang and Wu, 2008). In this study we use EEMD with 3000 ensemble members, when dealing with noisy input data.

\subsection{Synthetic Time Series}

We use a two-tone signal, comprised of two sinusoidal time series, to examine the ability of the EMD/EEMD method to resolve signals with similar frequencies. The use of synthetic data permits direct comparison of the IMFs obtained from EMD and EEMD with the known signal components. Specifically, we consider the superposition of two signals $S_{1}(t)$ (high frequency mode) and $S_{2}(t)$ (low frequency mode) defined as:

$$
S_{1}(t)=0.6 \sin \left(\frac{2 \pi t}{T_{1}}\right) ; \quad S_{2}(t)=0.6 \sin \left(\frac{2 \pi t}{T_{2}}\right) ; \quad t=1,2, \ldots, 1000 .
$$

$T_{1}$ and $T_{2}$ are the periods of the two component signals. We take $T_{1}=50$ as fixed, and allow $T_{2}$ to vary as $T_{2}=T_{1}+i, i=1,2, \ldots, 100$.

The synthetic two-tone input data is then defined as:

$$
y_{0}(t)=S_{1}(t)+S_{2}(t) ; \quad t=1,2, \ldots, 1000 .
$$

To emulate a noisy input time series, we add noise to the two-tone signal $y_{0}(t)$ :

$$
y_{1}(t)=y_{0}(t)+\epsilon(t) ; \quad t=1,2, \ldots, 1000,
$$

where $\epsilon \sim N(0, \delta)$ and $\delta$ is taken as $20 \%$ of the standard deviation of $y_{0}$. Figure 1 shows four examples of the two-tone input signals used in the study.

\section{RESUlts AND Discussion}

In order to address the performance of the method we consider the mean absolute error (MAE) of the IMFs extracted using EMD/EEMD:

$$
\mathrm{MAE}_{j}=\frac{\sum_{t=1}^{1000}\left|S_{j}(t)-\mathrm{IMF}_{j}(t)\right|}{1000} ; j=1,2
$$


F.M. Ziaeyan Bahri and J.J. Sharples, EMD and the two-tone separation problem with noise.
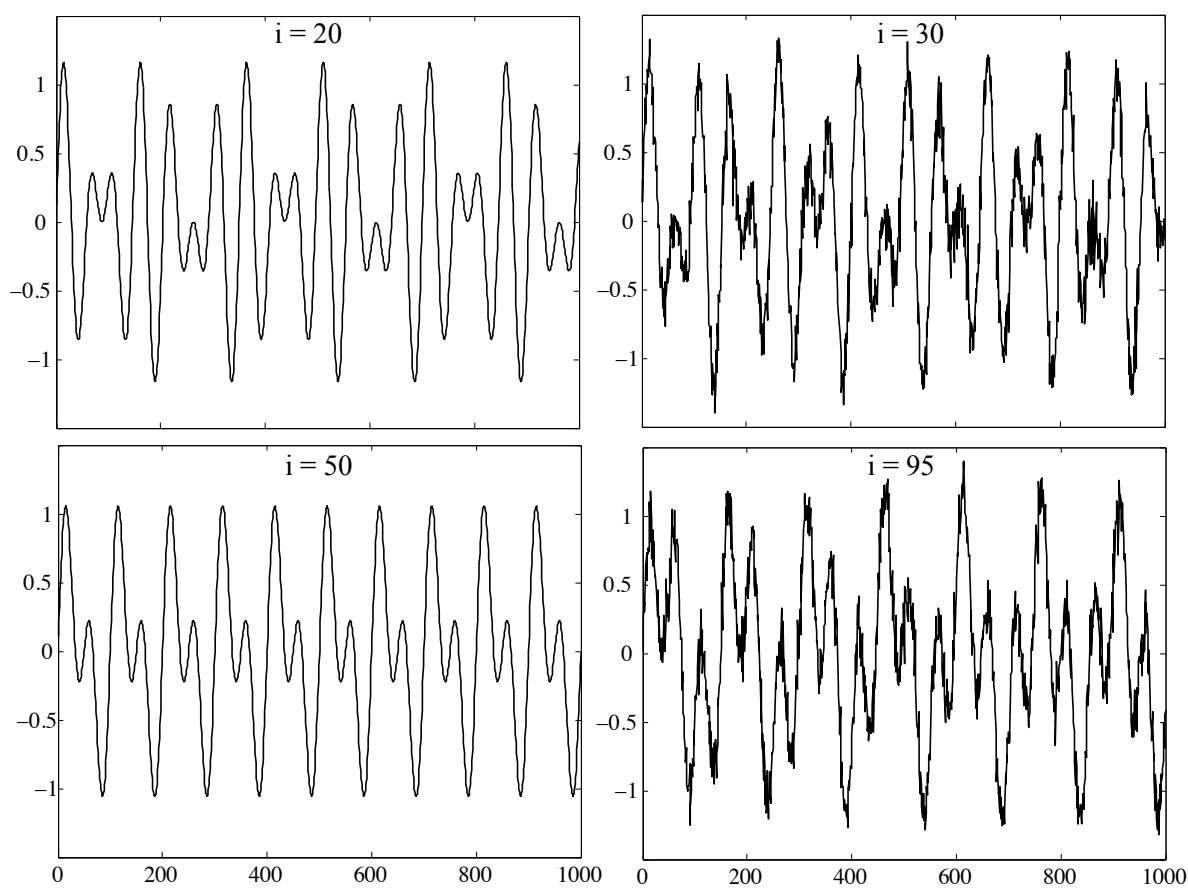

Figure 1. Examples of two-tone input signals used in the study. Left panels: noiseless data $y_{0}$. Right panels: noisy data $y_{1}$.

\subsection{Two-tone signal in the absence of noise}

Figure 2 shows the results of applying EMD to the noiseless two-tone signal. The left panels show how MAE 1 (left) and $\mathrm{MAE}_{2}$ (right) vary with $i$, while the remaining panels provide examples of how the extracted IMFs compare with the actual high frequency (left) and low frequency (right) components for different representative values of $i$.

The response of MAE $\mathrm{E}_{1}$ to increasing $i$ in Figure 2 indicates that EMD is not able to resolve the signal $S_{1}$ when $i \lesssim 20$. This can be seen in the middle left panel of Figure 2, which shows when $i=20$ the extracted high frequency IMF (red curve) bears a poor resemblance to the actual component. Figure 2 indicates that $\mathrm{MAE}_{1}$ decreases with $i$ in the range $20 \lesssim i \lesssim 50$ after which it levels out. The bottom left panel of Figure 2 shows how the extracted $\mathrm{IMF}_{1}$ compares to the actual component for the case $i=50$; a very good fit is evident.

The panels on the right of Figure 2 show how EMD performs in extracting the low frequency component $S_{2}$. The response of $\mathrm{MAE}_{2}$ to variation in $i$ exhibits almost identical behaviour to $\mathrm{MAE}_{1}$.

We note that visual assessment of the results suggested that the separate signals $S_{1}$ and $S_{2}$ could be adequately discerned when $\mathrm{MAE}_{1,2} \lesssim 0.2$. For $0.2 \lesssim \mathrm{MAE}_{1,2} \lesssim 0.3$ the extracted IMFs were marginally distinguishable but exhibited a degree of mode-mixing. When $\mathrm{MAE}_{1,2} \gtrsim 0.3$ it was not possible to visually distinguish the two component signals in the extracted IMFs.

We note that $i=20$ corresponds to a signal frequency ratio of $f \approx 0.71$ and so these results are broadly consistent with the results obtained by Rilling and Flandrin (2008) for component signals with an amplitude ratio of $a=1$. Indeed, they found that EMD could identify a two-tone signal for $f \gtrsim 0.75$, and they found that the two component signals could be successfully separated below a signal frequency ratio of $f \approx 0.55$. In the present study this value of $f$ corresponds to a value of $i \approx 40$ and $\mathrm{MAE}_{1,2} \approx 0.1$.

\subsection{Two-tone signal with noise}

Figure 3 shows the results of applying EEMD to the noisy two-tone signal $y_{1}$. The top left panel of Figure 3 shows the response of $\mathrm{MAE}_{1}$ to changes in $i$. The results again indicate that EEMD does a poor job of resolving the two component signals when $i \lesssim 20-25$. In comparison to the noiseless case, the reduction in $\mathrm{MAE}_{1}$ for $i>20$ is more gradual in Figure 3. Furthermore, for the noisy two-tone case, MAE 1 does not start to flatten out until $i \gtrsim 60$, and displays considerable variability. 
F.M. Ziaeyan Bahri and J.J. Sharples, EMD and the two-tone separation problem with noise.
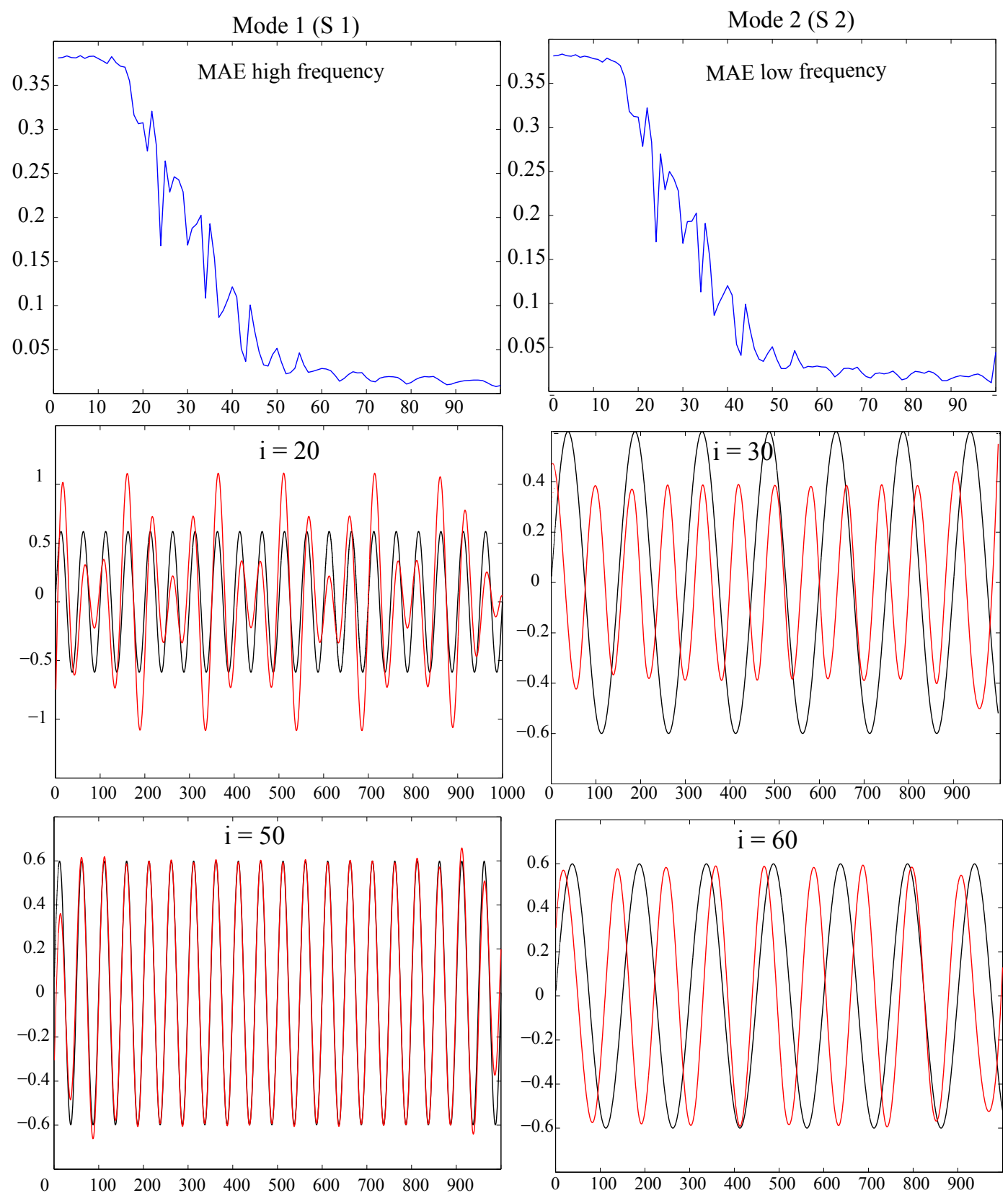

Figure 2. Results of EMD applied to the noiseless two-tone signal $y_{0}$. The two top panels show MAE 1,2 . The remaining panels show examples of how the extracted IMFs (red) compare to the actual signal components (black) for particular values of $i$.

The middle left panel of Figure 3 shows that for $i=20$, EEMD applied to the noisy input signal gave similar results for $\mathrm{IMF}_{1}$ as did EMD applied to the noiseless signal. The bottom left panel of Figure 3 shows that in the case of the noisy input signal, EEMD is still having difficulty resolving the high frequency component when $i=50$, compared to the noiseless case.

The panels on the right of Figure 3 show how EEMD performs at extracting the low frequency component $S_{2}$ from the noisy input signal. Similar to the other cases, $\mathrm{MAE}_{2}$ is relatively constant up until $i \approx 20$ and then begins to decrease up to $i \approx 65-70$, after which it levels off. The middle right and bottom right panels of Figure 3 provide examples of how well the extracted $\mathrm{IMF}_{2}$ compares to the low frequency component $S_{2}$. It can be seen that even when $i=95$, the extracted mode is not in perfect agreement with the actual component.

It is worth noting that the value $i=20-25$ corresponds to a frequency ratio of $f \approx 0.66-0.70$. This value is slightly lower that the threshold value of $f \approx 0.75$ reported by Rilling and Flandrin (2008), where EMD 
F.M. Ziaeyan Bahri and J.J. Sharples, EMD and the two-tone separation problem with noise.
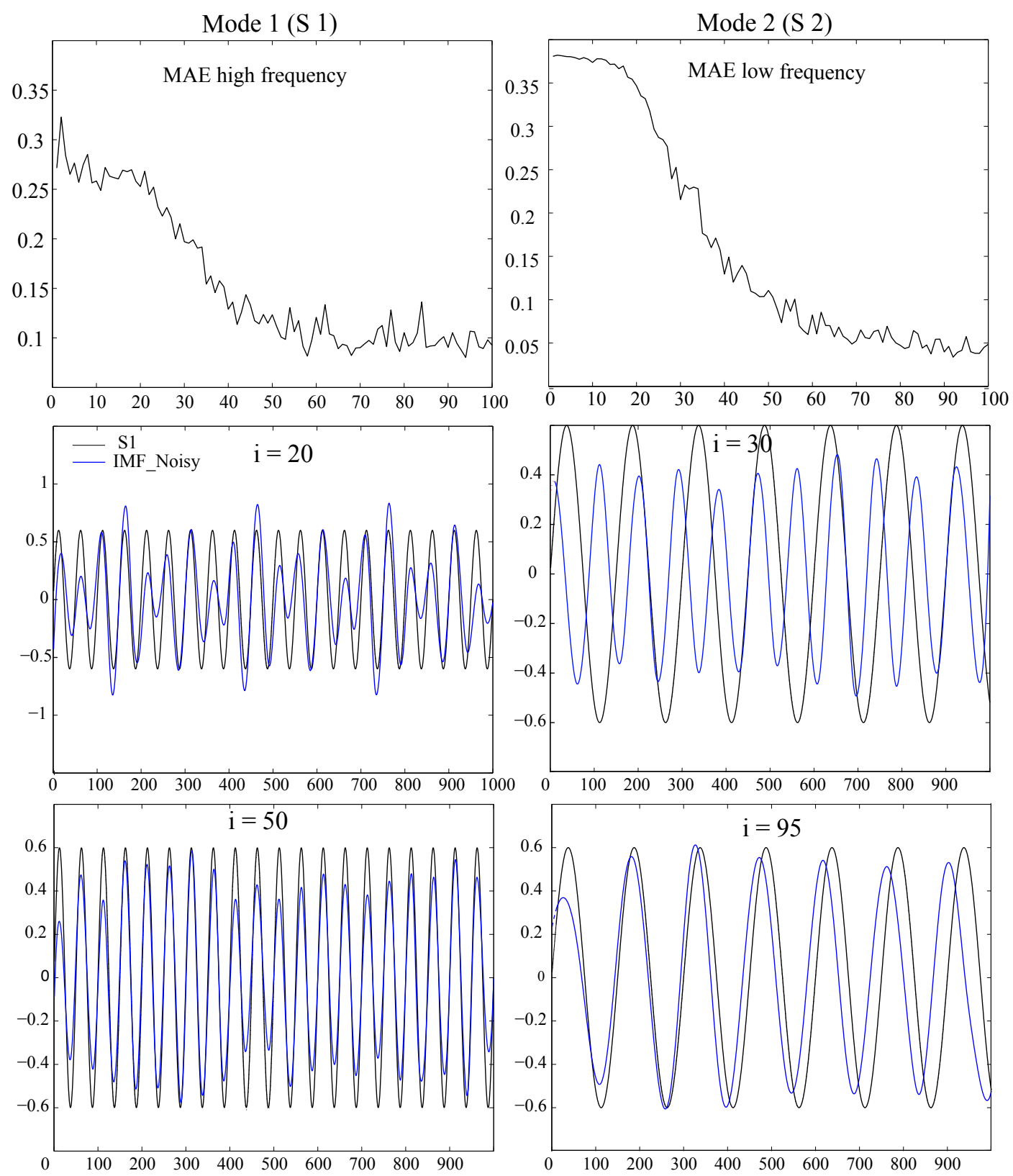

Figure 3. Results of EEMD applied to the noisy two-tone signal $y_{1}$. The two top panels show MAE ${ }_{1,2}$. The remaining panels show examples of how the extracted IMFs (blue) compare to the actual signal components (black) for particular values of $i$.

either considers the two-tone signal as a single waveform, or produces ambiguous results. Our findings are therefore roughly consistent with those of (Rilling and Flandrin, 2008), but suggest that the addition of noise to the input signal slightly diminishes the ability of EEMD to correctly identify the input signal as a two-tone signal. While there needs to be more investigation of the noisy two-tone separation problem using different noise profiles, our results suggest that EEMD cannot successfully separate the two tone signal if the frequency ratio is above about $f=0.7$.

For the noisy input signal, it was found that minimal $\mathrm{MAE}_{1,2}$ values were attained for $i \gtrsim 60-70$, compared to $i \gtrsim 50$ for the noiseless case. In terms of the frequency ratio, $i \approx 60-70$ corresponds to $f \approx 0.40-0.45$, which is less than the $f \approx 0.55$ found by (Rilling and Flandrin, 2008) as the threshold value, below which EMD can successfully resolve the two sinusoidal components. Thus, while the addition of noise reduces the threshold above which EEMD treats the two-tone signal as a single waveform only by about 7\%, it has a more 
F.M. Ziaeyan Bahri and J.J. Sharples, EMD and the two-tone separation problem with noise.

pronounced effect on the ability of EEMD to successfully separate the two component signals. The threshold value for the frequency ratio in this case is reduced by about $27 \%$.

Assessing the performance of EEMD visually, it was found that for $i \lesssim 25$ EEMD could not distinguish the two signals, while results were intermittent for $i$ between 25 and 60. EEMD was able to distinguish the two signals for $i \gtrsim 60$, but the modes were not accurately identified until $i \gtrsim 95$ for the noisy input two-tone signal. In presence of noise, more mode-mixing was evident across the modes; it is possible that this could be improved using alternate interpolation algorithms as the basis for EEMD, such as smoothing splines instead of cubic splines. This will be considered in future work.

\section{Conclusion}

This study considered the two-tone separation problem using EMD and EEMD in the presence of noise. Results from using EMD on noiseless two-tone signals were in general agreement with previous studies. It was found, however, that the presence of noise has an effect on the ability of EEMD to successfully identify and separate the two component signals. In particular, EEMD could not distinguish a noisy two-tone signal from a single waveform if the frequency ratio of the two component signals was above $f \approx 0.7$. Moreover, EEMD could only successfully identify the two component signals when the frequency ratio was below about $f=0.4$.

The present study did not consider the effect of adjusting the amplitude of the component signals relative to one another. This will be investigated in future work, as will the effects of increasing the amplitude and non-stationarity of the noise added to the two-tone signal.

\section{ACKNOWLEDGMENTS}

F.M. Ziaeyan Bahri acknowledges the financial support of the School of Physical, Environmental and Mathematical Science, UNSW Canberra.

\section{REFERENCES}

Boudraa, A.-O. and J.-C. Cexus (2007). EMD-based signal filtering. Instrumentation and Measurement, IEEE Transactions on 56(6), 2196-2202.

Bracewell, R. (1965). The Fourier Transform and IIS Applications. New York.

Donnelly, D. (2006). The Fast Fourier and Hilbert-Huang transforms: a comparison. In Computational Engineering in Systems Applications, IMACS Multiconference on, Volume 1, pp. 84-88. IEEE.

Feldman, M. (2009). Analytical basics of the EMD: Two harmonics decomposition. Mechanical Systems and Signal Processing 23(7), 2059-2071.

Huang, N. E., Z. Shen, S. R. Long, M. C. Wu, H. H. Shih, Q. Zheng, N.-C. Yen, C. C. Tung, and H. H. Liu (1998). The empirical mode decomposition and the Hilbert spectrum for nonlinear and non-stationary time series analysis. In Proceedings of the Royal Society of London A: Mathematical, Physical and Engineering Sciences, Volume 454, pp. 903-995. The Royal Society.

Huang, N. E. and Z. Wu (2008). A review on Hilbert-Huang transform: Method and its applications to geophysical studies. Reviews of Geophysics 46(2).

Kolláth, Z. and K. Oláh (2009). Multiple and changing cycles of active stars-I. Methods of analysis and application to the solar cycles. Astronomy \& Astrophysics 501(2), 695-702.

Rilling, G. and P. Flandrin (2008). One or two frequencies? the empirical mode decomposition answers. IEEE transactions on signal processing 56(1), 85-95.

Schlurmann, T. (2002). Spectral analysis of nonlinear water waves based on the Hilbert-Huang transformation. Transactions-American Society of Mechanical Engineers Journal of Offshore Mechanics and Arctic Engineering 124(1), 22-27.

Wickerhauser, M. V. (1994). Adapted wavelet analysis from theory to software. IEEE press.

Wu, Z., N. E. Huang, and X. Chen (2011). Some considerations on physical analysis of data. Advances in Adaptive Data Analysis 3(01n02), 95-113. 